\title{
Praktiken modellieren: Dialogmodellierung als Methode der Interaktionalen Linguistik
}

\begin{abstract}
Der Beitrag plädiert dafür, die Interaktionale Linguistik stärker für modellorientierte Forschung und datengeleitete Methoden zu öffnen. Er stellt eine Methode vor, wie auf der Basis von Korpora datengeleitet Praktiken rekonstruiert und modelliert werden können. Ausgehend von einer Diskussion der tiefgreifenden Veränderungen, die die Digitalisierung für die Linguistik mit sich bringt, und einer Auseinandersetzung mit dem Modellbegriff, wird der Begriff der (Kommunikativen) Praktik in Abgrenzung zum Begriff der Kommunikativen Gattung bestimmt. Im Anschluss wird am Beispiel von Trostdialogen in OnlineForen eine korpusgeleitete Methode zur Dialogmodellierung vorgestellt. Schließlich werden die Folgen der menschlichen Interaktion mit maschinellen Dialogsystemen reflektiert.
\end{abstract}

\section{Das „imitation game“: Das Missverständnis mit ELIZA}

„It is said that to explain is to explain away“ (Weizenbaum 1966, S. 36). Mit diesen Worten eröffnet Joseph Weizenbaum seinen 1966 erschienen Aufsatz „ELIZA - a computer program for the study of natural language communication between man and machine“. Darin erläutert Weizenbaum die Funktionsweise eines Computerprogramms, das es den Benutzerinnen und Benutzern erlaubte, in natürlicher Sprache mit dem Computer zu interagieren; und zwar als eine von drei Spielarten in der Rolle eines Patienten im Gespräch mit DOCTOR, der Simulation eines Psychotherapeuten, der die non-direktiven Methoden der klientenzentrierten Psychotherapie nach Carl Rogers anzuwenden schien. Und dies mit großem Erfolg: Kopien des Programms verbreiteten sich äußerst schnell und ELIZA avancierte zu einem beliebten Schauobjekt, mit dem das Potenzial von Informationstechnologien verständlich für jedermann demonstriert werden konnte. Die Faszination, die von ELIZA ausging, erfasste nicht nur die neugierige Öffentlichkeit, sondern beflügelte auch Wissenschaftlerinnen und Wissenschaftler. So sahen einige praktizierende Psychiater in DOCTOR ein zwar noch nicht vollständiges, aber doch avanciertes Modell ihrer selbst: 
A human therapist can be viewed as an information processor and decision maker with a set of decision rules which are closely linked to short-range and long-range goals. [...] He is guided in these decisions by rough empiric rules telling him what is appropriate to say and not to say in certain contexts. (Colby/Watt/Gilbert 1966, S. 151)

Für andere symbolisierte ELIZA die Lösung eines kaum für lösbar gehaltenen Problems: dass Computer natürliche Sprache verstehen (Weizenbaum 1976, S. 7 f.).

Diese Interpretationen standen den Intentionen, die Weizenbaum bei der Entwicklung von ELIZA verfolgt hatte, diametral entgegen. Weder hatte er ein Modell einer psychiatrischen Methode formulieren, noch hatte er eine Lösung für das Verstehen natürlicher Sprache durch Computer vorlegen wollen. Vielmehr hatte er die Absicht zu zeigen, dass Sprache gerade nicht kontextlos verstanden werden kann. In seinem späteren Buch „Computer Power and Human Reason“ stellte er klar: „I had tried to say [...] that language is understood only in contextual frameworks, that even these can be shared by people to only a limited extent, and that consequently even people are not embodiments of any such general solution" (Weizenbaum 1976, S. 7). Er machte Technologien wie ELIZA gar dafür verantwortlich, ein Bild des Menschen als berechenbare Maschine zu verbreiten. Dieses Bild verdanke sich freilich nicht den Technologien selbst, sondern ihrer Rezeption durch eine Öffentlichkeit, die das Funktionieren dieser Technologien nicht durchschaue. Um ELIZA ihre Magie und Aura zu nehmen, um das Programm verstehbar zu machen und es damit als simple Maschine zu demaskieren, schrieb er den eingangs erwähnten Aufsatz. „The object of this paper is to cause [...] a re-evaluation of the program about to be ,explained“" (Weizenbaum 1966, S 36).

Weizenbaum wurde also missverstanden und wird es noch heute: Er, der Skeptiker im Hinblick auf die Sprach- und vor allem Kommunikationsfähigkeit des Computers, wird noch heute in jeder Abhandlung zur Dialogmodellierung als wichtiger Vordenker auf dem Weg zur Touring-Vollständigkeit ${ }^{1}$ geführt. Die Tatsache, dass viele Menschen bei der Interaktion mit DOCTOR über viele Turns hinweg das Gefühl hatten, mit einer Person zu interagieren, brachte das Programm nämlich für die Zeitgenossen in die Nähe der Turing-Vollständigkeit wogegen sich Weizenbaum ausdrücklich verwahrte (vgl. ebd., S. 42 f.). Doch auch wenn ELIZA kein vollständiges Modell menschlicher Kommunikation und kein proof of concept für die Verstehensfähigkeit des Computers ist, ist es in anderer Hinsicht doch ein gelungenes Modell bestimmter Dimensionen humaner Kommunikation.

1 Der Terminus ,Touring-Vollständigkeit' bezeichnet die Eigenschaft eines logischen Systems, alle Funktionen berechnen zu können, die eine Touring-Maschine berechnen kann. Das System ist dann eine Emulation der Maschine, an die in der Dialogmodellierung ein idealer Sprecher/Hörer tritt. 
ELIZA gelingt es, beim Benutzer bzw. der Nutzerin die Fiktion aufrecht zu erhalten, sie würden von DOCTOR verstanden. Dafür bedient sich das Programm einer speziellen Interaktionsmodalität, der non-direktiven Methode der klientenzentrierten Psychotherapie:

This mode of conversation was chosen because the psychiatric interview is one of the few examples of categorized dyadic natural language communication in which one of the participating pair is free to assume the pose of knowing almost nothing of the real world. (Weizenbaum 1966, S. 42)

DOCTOR kann sich darauf beschränken, Fragen zu stellen bzw. über Schlagwörter Themen zu setzen, über die der Benutzer sprechen soll, ohne durch seine Äußerungen ein vertieftes Wissen, eine Beurteilung oder eine emotionale Beteiligung im Hinblick auf den verhandelten Sachverhalt zum Ausdruck zu bringen. Die kommunikative Gattung, die die Art der Gesprächsführung kontextualisiert, ermöglicht für den menschlichen Interaktionspartner wiederum die Projektion, es mit einem kompetenten, geschulten Gegenüber zu tun zu haben. Dass die Fiktion, ein kohärentes Gespräch zu führen, über mehrere Turns aufrecht erhalten werden kann, liegt freilich nicht an den Qualitäten des Computerprogramms allein, sondern vor allem an den Leistungen, die die Nutzer bzw. Nutzerinnen erbringen. Aus den Äußerungen von DOCTOR ziehen sie Schlussfolgerungen, ohne dass diese Schlüsse explizit kodiert wären. Auch wenn Konversationsmaximen verletzt werden, machen sie Inferenzen auf Implikaturen, die den Äußerungen von DOCTOR Sinn unterstellen. ELIZA ist damit lesbar als ein algorithmisches System, das das Funktionieren bestimmter universalpragmatischer Aspekte sprachlicher Interaktion modelliert. Als Modell hat es zwar nur begrenzte Reichweite, gleichwohl erlaubt es aber Simulationen natürlichsprachlicher Kommunikation. Mit Hilfe dieser Simulationen sind beispielsweise Analysen zum Scheitern von Kommunikation möglich, die Rückschlüsse auf die Konstitutionsbedingungen und Grenzen von Inferenzen auf konversationelle Implikaturen erlauben.

Weizenbaums Tragik liegt also darin, ein Modell zur Demonstration der Unzulänglichkeit maschineller Sprachproduktion geschaffen zu haben, das zusammen mit der antizipierten Reaktion der Interaktionspartner auf die Benutzer von ELIZA wie eine gelungene Imitation, ein funktionierendes Modell menschlicher Kommunikation wirkte.

Offenbar war es mit so einfachen Mitteln, wie sie Weizenbaum einsetzte, möglich, menschliche Kommunikation zu modellieren. Und die Verblüffung, die ELIZA auslöste, verschwand nicht mit der Offenlegung des Codes und der Explizierung seiner Funktionsweise. Das Modell selbst geriet zum Faszinosum: Wie konnte es sein, dass mit Hilfe eines Computermodells fundamentale Einsichten 
in die Mechanismen sprachlicher Interaktion möglich waren? Weizenbaums Aufsatz jedenfalls hat das heuristische Potenzial, das sein Modell für das Verstehen von Interaktion hat, nicht „wegerklärt“.

Der vorliegende Beitrag beschäftigt sich mit der Frage, was heute - im Zeitalter von Digitalisierung, Big Data und sehr viel leistungsfähigeren Rechnern, als sie Weizenbaum zur Verfügung hatte - die Beschäftigung mit computergenerierten Modellen von Sprache und Interaktion zu einem tieferen Verständnis menschlicher Kommunikation beitragen kann. Er regt an, Fortschritte im Bereich der Dialogmodellierung, die in Computerlinguistik, Angewandter Informatik und den Ingenieurswissenschaften (speziell der Mensch-Maschine-Interaktion) gemacht wurden, in eine Methode jener sprachwissenschaftlichen Paradigmen $\mathrm{zu}$ wenden, die verbale Interaktion vor allem qualitativ betreiben, namentlich die Dialoganalyse, die ethnografische Gesprächsanalyse und die Konversationsanalyse, die ich im Folgenden vereinfachend unter der Bezeichnung „Interaktionale Linguistik“ zusammenfasse. Die Bezeichnung „Dialogmodellierung“ (Jokinen 2009) verwende ich dagegen für informatisch-ingenieurswissenschaftliche Ansätze, die mit dem Ziel entwickelt wurden, die Interaktion zwischen Mensch und Maschine zu optimieren.

Um die Möglichkeiten der Anwendung der Dialogmodellierung in der Interaktionalen Linguistik genauer bestimmen zu können, sollen zunächst die im Zuge der digitalen Revolution sich dramatisch verändernden Bedingungen des Forschens reflektiert werden; und zwar im Hinblick auf die Entwicklung einer digitalen Forschungslogik einerseits, aber auch im Hinblick auf die Gefahren, die eine theorieabgewandte Angewandte Informatik für die Kultur- und Geisteswissenschaften mit sich bringt (Abschnitt 2). Im Anschluss soll das Arbeiten in Modellen als eine spezielle Form des Verstehens wissenschaftlicher Gegenstände profiliert werden (Abschnitt 3), ehe ausgehend von einer Diskussion des Praktikenbegriffs (Beispiel 4) am Beispiel von Trostdialogen in Internetforen das heuristische Potenzial von Dialogmodellierung und Dialogsimulation illustriert werden sollen (Abschnitt 5).

\section{Interaktionale Linguistik im digitalen Zeitalter und das Ende vom „Ende der Theorie“}

Die Beschäftigung mit verbaler Interaktion erfolgt in der Linguistik vorwiegend aus konversations- und gesprächsanalytischer Perspektive. Und dies mit gutem Grund: Will man etwa die konstitutiven Eigenschaften von Gesprächen, ihre Konstitutivität, Prozessualität, Interaktivität, Methodizität und Pragmatizität (vgl. Deppermann 2008, S. 8 f.) nur annähernd adäquat in der Analyse berücksichtigen, müssen kontextsensitive, die Sequenzierung systematisch einbeziehende, 
emische Kategorien berücksichtigende Analyseverfahren zum Einsatz kommen. Gesprächsanalyse betreiben heißt nach Deppermann, Gesprächspraktiken zu explizieren und zwar auf der Basis einer materialgestützten Entwicklung von Fragestellungen und falladäquaten Aussagen unter Verwendung authentischen Materials (vgl. ebd., S. 11). Die Analyse des Einzelfalls behandelt die Interaktion als sich selbst interpretierenden Vorgang (Hausendorf 1997, S. 269) und dient primär der Erweiterung des Verstehenshorizonts, der Verfeinerung von Beschreibungskategorien und der Erweiterung des Wissens um Praktiken der Wirklichkeitskonstitution durch verbale und nonverbale Interaktion. Die aus den Analysen gewonnenen theoretischen Erkenntnisse versprechen aufgrund der Tatsache, dass sie nicht anhand des Testens vorgängiger Hypothesen gewonnen, sondern streng aus den analysierten Daten heraus generiert werden, ein hohes Maß an Gegenstandsadäquatheit und praktischer Relevanz.

Ich möchte in diesem Beitrag dennoch dafür plädieren, dass sich die Linguistik für andere Methoden der Beschäftigung mit verbaler Interaktion verstärkt öffnet, insbesondere deshalb, weil die bisherigen Methoden der interaktionalen Linguistik nur eingeschränkt geeignet sind, das Potenzial, das die Digitalisierung für die Sprachwissenschaft bereitstellt, produktiv zu nutzen. Das soll freilich nicht heißen, dass die Interaktionale Linguistik sich nicht auch für neue Technologien öffnet. Allerdings geschieht dies eher im Sinn einer Digitalisierung ihrer Apparate und Methoden als im Sinne einer Wendung hin $\mathrm{zu}$ einer digitalen Forschungslogik (analog zur Unterscheidung von „digitized“ vs. „digital“). Die Digitalisierung, die im Folgenden in dreierlei Hinsicht spezifiziert werden soll, verändert die Bedingungen sprachwissenschaftlicher Forschung dramatisch:

1. Die Verdatung der Welt: Immer mehr Informationen werden in ein digitales Format gebracht oder schon in digitaler Form produziert. „Digital“ bedeutet „abzählbar sein“, d.h. dass Informationen in eine numerische Form gebracht werden. Parallel zur Entstehung von „Big Data“ ermöglicht die Digitalisierung damit auch

2. die Zusammenführung und damit kombinierte Analyse von Daten unterschiedlichster Provenienz: Die Repräsentation unterschiedlichster Informationstypen in einem numerischen Modell macht es möglich, unterschiedlichste Informationen durch Algorithmen miteinander zu verknüpfen und zu analysieren. Hinzu kommen

3. Fortschritte in den Verfahren maschinellen Lernens: Sie ermöglichen es, datengeleitet Muster in großen Datenmengen aufzudecken, die neben dem analytischen Nutzen und dem Befragen traditioneller Kategorien auch Vorhersagen und pragmatisch-normative Simulationen erlauben. Für diese Entwicklung habe ich zusammen mit Kollegen die Bezeichnung, data-driven Turn' geprägt (vgl. Scharloth/Eugster/Bubenhofer 2013). 
Für die traditionelle Gesprächsanalyse werden analoge Daten verbaler Interaktion durch Digitalisierungstechnologien schneller und kompletter (bspw. multimodal) erfassbar und können im Hinblick auf bestimmte Parameter in großen Mengen analysiert werden. Noch mehr gilt dies freilich für Interaktionsdaten, die im Medium des Digitalen entstanden sind (,born digital“). Chats, Meldungen aus Kurznachrichtendiensten, ganze Online-Diskussionsforen stehen in unermesslicher Menge zur Verfügung und sind im Hinblick auf die technischen Kontexte ihrer Entstehung in vielen Fällen annähernd vollständig dokumentierbar. Gegen diese Fülle an Daten nehmen sich die existierenden Korpora gesprochener Sprache eher bescheiden aus, nicht nur im Hinblick auf den Umfang, sondern auch im Hinblick auf die durch sie in befriedigendem Umfang abgedeckten Sprachhandlungen, Praktiken und Gesprächsthemen. Um die speziellen Vorzüge digitaler Daten für die Linguistik fruchtbar zu machen, sind technische Fertigkeiten, wie sie von Korpus- und Computerlinguistik sowie der Informatik entwickelt wurden, von Nöten - hierzu später mehr. Denn so positiv diese Entwicklungen auf den ersten Blick auch sind, so stellen sie doch auf lange Sicht auch eine Bedrohung für eine eigenständige, den Traditionen und Erkenntnisinteressen der eigenen Disziplin verpflichteten digitalen Interaktionalen Linguistik dar.

Das Bedürfnis von Nutzerinnen und Nutzern nach natürlichsprachlicher Interaktion mit Computern hat dazu geführt, dass sowohl die Informatik als auch die IT-Industrie ein virulentes Interesse an Dialogsystemen entwickelt haben. Diese Bereiche des Forschens werden teilweise von einem Denkstil geprägt, der für alternative Ansätze keinen Raum lässt. Exemplarisch lässt sich dies an einem programmatischen Essay von Chris Anderson (2008), dem Chefredakteur des „Wired Magazine“, zeigen. In dem „The End of Theory“ betitelten Aufsatz verkündete er angesichts der Datensintflut das Ende wissenschaftlicher Methoden. Das Anhäufen großer Datenschätze und die Möglichkeit ihrer effizienten Analyse, also „faced with massive data, this approach to science - hypothesize, model, test is becoming obsolete“. Das empirisch-analytische Wissenschaftsbild ist nach Meinung Andersons obsolet geworden, weil die Zahlen für sich selbst sprechen:

Out with every theory of human behavior, from linguistics to sociology. Forget taxonomy, ontology, and psychology. Who knows why people do what they do? The point is they do it, and we can track and measure it with unprecedented fidelity. With enough data, the numbers speak for themselves.

Für die Mensch-Maschine-Kommunikation würde dies bedeuten: Es bedarf keiner linguistischen Theorie mehr, um eine natürlichsprachliche Interaktion zwischen Menschen und Computern zu ermöglichen. Linguistische Theorie wird ersetzt durch hochgradig ausdifferenzierte wahrscheinlichkeitsbasierte Kalküle, die 
als Ergebnis der Analyse großer Datenmengen anfallen. Dass auf eine Äußerung X Äußerung Y erfolgen kann oder muss, ergibt sich dann aus der in der jeweiligen Kommunikationssituation vorhandenen Merkmalskonstellation, die in den Trainingsdaten in gleicher oder ähnlicher Weise auch zu Äußerung Y geführt hat. Interaktion ist demnach berechenbar, wenn für das System nur genügend Trainingsdaten zur Verfügung stehen. Das Ende der Theorie brächte auch die Linguistik in Rechtfertigungszwänge: Wozu braucht man noch eine Interaktionale Linguistik, wenn man ein stochastisches Modell von verbaler Interaktion hat, das Computer in die Lage versetzt, wie Menschen zu kommunizieren?

Es ist freilich offensichtlich, dass Andersons Utopie von der theoriefreien Welt auf den Implikationen der Wendung ,[w]ith enough data, the numbers speak for themselves“ beruht und mit ihr zum Scheitern verurteilt ist. Sie birgt nämlich einerseits das Potenzial, kritikimmunisierende ad-hoc-Modifikationen (Popper 1966, S. 57 f.) zu generieren, denn wenn ein stochastisches Simulationsmodell nicht funktioniert, dann könnte immer eingewendet werden, es hätten nicht genügend Daten vorgelegen. Andererseits verdankt die Wendung „with enough data, the numbers speak for themselves“ ihre vermeintliche Plausibilität einem unterkomplexen „data“-Begriff. Denn Daten sind durch Messung oder Beobachtung zustande gekommene Repräsentationen, die in irgend einer Form strukturiert sein müssen, um mit Hilfe von Codes als Informationen lesbar zu sein. Keine Beobachtung jedoch kommt ohne Theorie aus, keine Strukturierung ohne begrifflich-theoretische Kategorisierung. „Es gibt keine reinen Daten“ (Stachowiak 1973, S. 288). Um verbale Interaktion messbar zu machen, müssen beispielsweise theorieförmige Annahmen darüber gemacht werden, was Wörter, Sätze oder Turns sind. In die Bestimmung dessen, was überhaupt gemessen werden soll, fließt also theorieförmiges Wissen ein. Nur wenn überhaupt relevante Merkmale erfasst werden, können die Ergebnisse datengeleiteter Analysen valide sein. Ohne eine theoriegeleitete Bestimmung relevanter Merkmale wäre nämlich nicht entscheidbar, ob es sich bei einem datengeleitet generierten Modell um ein Artefakt handelt oder ob es sich gar Hintergrundvariablen verdankt, die nur durch eine vertiefte Kenntnis des Gegenstands aufgedeckt werden könnten. Es ist also nicht die Theorie, die in der Datensintflut ertrinkt, sondern es sind InternetTheoretiker wie Anderson.

Wenn im Folgenden Überlegungen zu einer datengeleiteten Modellierung von Kommunikativen Praktiken angestellt werden sollen, dann im Bewusstsein dessen, dass ohne Theorie auch maschinelle datengetriebene Ansätze keine befriedigenden Ergebnisse generieren können. Gleichwohl halte ich datengeleitete Ansätze für besonders geeignet, die oben skizzierten Effekte der Digitalisierung für die Linguistik zu nutzen, zumal sie sich an eine Tradition der Korpuslinguistik anschließen lassen, die sich als korpusgeleitet bezeichnen lässt. Im Gegensatz 
zum korpusbasierten Ansatz, in dem digitale Textkorpora der Überprüfung von Forschungshypothesen dienen, die unabhängig von der Analyse des Korpus entwickelt wurden, und die mittels bereits bewährter interpretativer linguistischer Analysekategorien an einem Korpus überprüft werden sollen, untersucht der datengeleitete Ansatz induktiv berechnete Muster in Korpora daraufhin, in welchem Verhältnis sie zu etablierten linguistischen Analysekategorien stehen und ob sich aus ihnen neue Hypothesen generieren lassen, und etabliert damit eine eigene „korpuslinguistische Perspektive“ (Perkhuhn/Belica 2006, S. 2). ${ }^{2}$ In Wolfgang Teuberts Skizze des datengeleiteten Paradigmas scheint sogar eine gewisse Ähnlichkeit zum Empirieverständnis der Gesprächsanalyse auf:

While corpus linguistics may make use of the categories of traditional linguistics, it does not take them for granted. It is the discourse itself, and not a language-external taxonomy of linguistic entities, which will have to provide the categories and classifications that are needed to answer a given research question. This is the corpus-driven approach. (Teubert 2005, S. 4)

Kommunikative Praktiken mittels datengeleiteter Methoden gegenstandsadäquat zu modellieren, bedeutet demnach, massenhafte Interaktionsdaten auf der Basis eines Begriffs von Kommunikativen Praktiken zu strukturieren, in dem so strukturierten Korpus induktiv Muster zu berechnen und diese Muster zu einem Modell zusammenzufassen. Dieses Modell freilich soll keine Black Box sein, wie Chris Anderson dies für theoriefreie Modelle als hinreichend empfand, vielmehr muss es selbst Gegenstand der Analyse werden können, um die algorithmisch gefundenen Zusammenhänge als linguistische re-interpretieren zu können.

\section{Modelltheoretische Vorüberlegungen}

Modelle werden in Wissenschaften typischerweise dann eingesetzt, wenn für Forschungsobjekte keine Beobachtungsdaten erhoben werden können (Ersatzfunktion), wenn die Durchführung von Experimenten zu teuer oder gefährlich

\footnotetext{
2 Für eine ausführliche Diskussion der Unterscheidung von corpus-based und corpus-driven vgl. Bubenhofer (2009, S. 99 ff.). Das corpus-driven-Paradigma geht auf Überlegungen bei Sinclair (1991) zurück und wurde dann von Tognini-Bonelli (2001, S. 65 ff.) ausformuliert. Im deutschsprachigen Raum wurde es z.B. in den Arbeiten von Kathrin Steyer (2004; Steyer/Lauer 2007; Steyer/Brunner 2009) aufgegriffen, die auf Konzepte und Methoden von Cyril Belica, Rainer Perkuhn, Holger Keibel und Marc Kupietz zurückgreift (Belica 1996, 2001; Perkuhn/Belica 2006).
} 
ist (Ersatzfunktion), wenn Prognosen als Werkzeuge der Planung und Entscheidungsfindung formuliert werden müssen (Vorbildfunktion), und schließlich als Vorarbeiten zu und pragmatischer Ersatz für komplexe, umfassende Theorien (Entwurfsfunktion). Im letzteren Sinn soll Modellieren auch im vorliegenden Beitrag betrieben werden.

Im Kern des Modellbegriffs steht nach Stachowiak (1983, S. 118) der Gedanke, dass Modelle immer Modelle von etwas sind, d.h. Repräsentationen eines Originals. Dennoch erfassen Modelle nur solche Eigenschaften des Originals, die für die Modellentwickler und/oder seine Verwender relevant sind.

Modelle [...] erfüllen ihre Ersetzungsfunktion stets a) für bestimmte Erkenntnis- und/oder Aktionssubjekte, b) innerhalb bestimmter Zeitintervalle der Originalrepräsentation und c) relativ zu bestimmten Zwecken und Zielen, denen die Modellbildung und die Modelloperationen unterliegen. (Stachowiak 1983, S. 118)

Auch wenn Stachowiaks Modellbegriff den Abbildcharakter stark zu betonen scheint, so war sich der Paderborner Philosoph doch darüber im Klaren, dass Modelle im Sinn des Neopragmatismus stets Konstruktionen sind. Modelle zeichnen sich demnach neben dem Bezug auf ein Original durch Subjekt-Bezüge (bspw. auf Schöpfer und Nutzer des Modells), Raum-Zeit-Bezüge (etwa Dauer der Gültigkeit, Dymanik, Modell von Vergangenem oder Zukünftigem) und Funktionen (Abbild, Vorbild, Entwurf, Ersatz) aus. ${ }^{3}$

Der Prozess der Modellierung besteht aus den Operationen der Abgrenzung, also der Nichtberücksichtigung irrelevanter Original-Objekte, der Reduktion als dem Weglassen von Objektdetails, die dem pragmatischen Zweck nicht dienlich sind, der Dekomposition als eine Zerlegung in einzelne Segmente, der Aggregation, verstanden als eine Vereinigung von Segmenten zu einem Ganzen, und der Abstraktion, also der Begriffs- und Klassenbildung als einem Beitrag zur Theoretisierung des Forschungsgegenstands. Diese Operationen sind freilich immer gebunden an pragmatische Zwecke und theorieförmige Konzepte, die den mit Hilfe des Modells untersuchten Realitätsausschnitt begrifflich vorstrukturieren.

Ein Modell ist das Ergebnis einer Konstruktion eines Modellierers, der für Modellnutzer eine Repräsentation eines Originals zu einer Zeit als relevant mit Hilfe einer Sprache deklariert. Ein Modell setzt sich somit aus der Konstruktion des Modellierers, dem Modellnutzer, einem Original, der Zeit und einer Sprache zusammen. (Schütte 1998, S. 59)

3 Stachowiak (1973, S. 56): ,indem sie [die Erkenntnis, JS] auf das - passive oder aktive - Erfassen von etwas aus ist, vollzieht sie sich relativ zu bestimmten Subjekten, ferner selektiv - intentional selektierend und zentrierend - und in je zeitlicher Begrenzung ihres Original-Bezuges.“ 
Modellklassen lassen sich entlang folgender Dichotomien bilden: Ist das Modell statisch oder dynamisch (ordnet es Eigenschaften zu oder simuliert es Prozesse), deterministisch oder stochastisch (konstituiert durch eindeutige Regeln oder wahrscheinlichkeitsbasierte Kalküle), kontinuierlich oder diskret (stetig oder sprunghaft zu diskreten Zeitpunkten)?

Im Folgenden soll der Versuch unternommen werden, für einen Teilbereich verbaler Interaktion, eine bestimmte kommunikative Praktik, zunächst ein statisches, stochastisch informiertes Modell zu entwerfen, um im Anschluss die Frage $\mathrm{zu}$ diskutieren, was der heuristische Nutzen eines Simulationsmodells für die Interaktionale Linguistik sein könnte.

\section{Zum Begriff der (Kommunikativen) Praktik}

Der Begriff der Praktik verdankt den Arbeiten von Reckwitz (2003, 2008), in denen er den „Practice Turn“ (vgl. Schatzki/Knorr Cetina/von Savigny (Hg.) 2001) in den Sozialtheorien reflektiert, seine neuerliche Konjunktur im deutschsprachigen Raum. Reckwitz bestimmt die Besonderheit von Praktiken im Vergleich zu anderen kultur- und sozialtheoretischen Konzepten anhand dreier Eigenschaften: 1. Die Materialität sozialer Praktiken (Reckwitz 2003, S. 290 f.): Die Praxistheorie rehabilitiert den menschlichen ,Körper und die ,Artefakte“, indem sie zunächst betont, dass Praktiken „ein Umgang von Menschen mit ,Dingen““ sind. Der Körper ist nicht bloß Instrument, sondern die Kompetenz zum Vollzug der Praktik ist Teil des Körperseins. „Kompetenz“ verweist auf implizites, inkorporiertes und verkörpertes Wissen, „Vollzug“ auf die Performativität des Handelns. In ihrer Wahrnehmung als ,skillful performance“ wird die Praktik zu einem Medium sozialer Verständigung und Anerkennung. Gleichzeitig sind Praktiken häufig um Artefakte zentriert, deren sinnhafter Gebrauch entweder die Praktik selbst ist oder zumindest einen wichtigen Teil ausmacht. Diese Artefakte determinieren freilich nicht ihren Gebrauch, setzen ihm aber aufgrund ihrer materiellen Beschaffenheit Grenzen und damit auch dem Sinn ihres Gebrauchs.

2. Die implizite Logik der Praktiken (Reckwitz 2003, S. 291 ff.): Hier geht es vor allem um die Frage, wie souverän und rational Subjekte gedacht werden können. Aus praxeologischer Sicht nämlich werden Praktiken als Tätigkeit verstanden, die wissensbasiert im Sinn eines Könnens (,knowing how“) sind. Routinemäßig angemessenes Handeln im Sinn dieses Wissens schafft „eine entsprechende ,Sinnwelt““, in der „Gegenstände und Personen eine implizit gewusste Bedeutung besitzen“. Die praxeologische Perspektive schließt eine 
Orientierung an Normen und die Annahme von Intentionen zwar nicht aus, betont jedoch dagegen die Implizitheit des handlungsleitenden Wissens, das gerade kein explizierbares Aussagewissen (,knowing that“) ist. Statt also davon auszugehen, dass ein Akteur Intentionen hat oder Normen befolgt, geht die praxeologische Forschung davon aus, dass in das praktische Wissen Intentionen und Normen eingeschrieben sind, die durch den Vollzug der Praktik im Träger dieser Praktik aktiviert bzw. ihm oder ihr zugeschrieben werden. Reckwitz benutzt hierfür die Metapher des „Einrückens“ der Akteure:

Für die Praxistheorie ist es nicht die vorgebliche Intentionalität, sondern die wissensabhängige Routinisiertheit, die das einzelne ,Handeln“ ,anleitet'; dies schließt teleologische Elemente nicht aus, die Praxistheorie betrachtet diese jedoch nicht als explizite und diskrete ,Zwecke‘ oder ,Interessen', sondern als sozial konventionalisierte, implizite Motiv/Emotions-Komplexe, die einer Praktik inhärent sind, in die die einzelnen Akteure ,einrücken“ und die sie dann möglicherweise als ,individuelle Interessen“ umdefinieren. (Reckwitz 2003, S. 293)

3. Die Routinisiertheit und die Unberechenbarkeit der Praktiken (Reckwitz 2003, S. $294 \mathrm{ff}$.): Anders als traditionelle Handlungstheorien, die ein zweckrationales Subjekt voraussetzen, und in denen sich die Struktur der sozialen Welt aus einem normativen Konsens ergibt, geht die Praxistheorie davon aus, dass praktisches Wissen, sobald es vermittelt und inkorporiert ist, dazu tendiert, von den Akteuren ,immer wieder eingesetzt zu werden und repetitive Muster der Praxis“ hervorbringt. Trotz der Routinisiertheit sozialer Praktiken müssen diese doch je kontextspezifisch angepasst werden, was Raum für ,interpretative und methodische Unbestimmtheit, Ungewissheit und Agonalität“ (Reckwitz 2003, S. 294) gibt, in dem sich Innovationen über die reine Reproduktion hinaus ergeben können.

Praktiken in dieser Lesart müssen freilich nicht kommunikativer Natur und schon gar nicht interaktiv sein. Um diesen Praktikenbegriff für die Linguistik fruchtbar und analytisch fassbarer zu machen, erscheint es sinnvoll, ihn um Bestandteile der Bestimmung Kommunikativer Praktiken im Anschluss an Fiehler et al. (2004) zu ergänzen und zwar speziell um kommunikative Dimensionen und einen sprachwissenschaftlichen Musterbegriff. Vorausgeschickt sei freilich, dass der zweckrationale Charakter von Kommunikativen Praktiken, der sich in der Definition von Fiehler et al. (ebd., S. 99) als ,präformierte Verfahrensweisen, die gesellschaftlich zur Verfügung stehen, wenn bestimmte rekurrente Ziele oder Zwecke kommunikativ realisiert werden sollen“ findet, nicht deckungsgleich mit dem eben skizzierten praxistheoretischen Praktikenbegriff ist. Für die genannten Autorinnen und Autoren sind Kommunikative Praktiken ,abgrenzbare, eigenständige kom- 
munikative Einheiten, für die ihre Zweckbezogenheit und Vorgeformtheit konstitutiv sind und für die es gesellschaftlich auch Bezeichnungen gibt“"(ebd., S. 100). Mit dem Praktikenbegriff - hier kommen Fiehler et al. und Reckwitz überein - ist die Annahme verbunden, dass die kommunikative Praxis generell stark vorgeformt bzw. vorstrukturiert ist, was bedeutet, dass die Auswahl und Anordnung der einzelnen Elemente keine allzu große Variation aufweist (Fiehler et al. 2004, S. 104). Diese Annahme unterscheidet Fiehler et al. beispielsweise von den Theoretikern der Kommunikativen Gattung, die lediglich für Gattungen rigide Strukturen konstatieren, für die sonstige kommunikative Praxis hingegen kaum Vorstrukturierungen annehmen. Praktiken weisen für Fiehler et al. zudem eine Affinität zu sprachlichen Mustern auf: Sie setzen sich aus sprachlichen Mustern zusammen, die als sprachliche Oberflächenphänomene aber auch als Verkettungen bestimmter Sprachhandlungen auftreten können (ebd., S. 102).

Im vorliegenden Beitrag soll der Versuch unternommen werden, eine Praktik in digitalen Medien zu modellieren, nämlich den Trostdialog in einem OnlineDiskussionsforum zu Trennungen, in dem sich vor allem die Verlassenen Hilfe holen. Es geraten also einerseits Tätigkeiten in den Blick, die born digital sind, andererseits handelt es sich um mediatisierte Praktiken. Mit Blick auf die im Anschluss an Reckwitz entwickelten Eigenschaften von Praktiken ist davon die Materialität in sehr spezifischer Weise berührt. Die Körper (und mit ihm somatische Dimensionen des verbalen Geschehens) werden unsichtbar zugunsten eines technisch entwickelten Mediums, in dem es (im konkreten Fall) nur sehr wenige semiotische Praktiken zur Inszenierung von Körperlichkeit jenseits des geschriebenen Wortes gibt. Zwar ließen sich (künftig) körperliche, visuelle und physiologische Aspekte des Schreibens durchaus erfassen und mit korpuslinguistischen Daten verknüpfen (die Anzahl und Qualität der Sensoren nimmt stetig zu), dennoch wäre der Nutzen solcher Daten im vorliegenden Fall fragwürdig: Denn weil der Körper von der sozialen Umwelt nicht (oder bestenfalls in mediatisierter Form) wahrgenommen werden kann, kann er nicht im Sinne einer ,skillful performance' interpretiert und zum Teil der Praktik werden (Reckwitz 2003, S. 290). Auch die Artefakte, um die die Praktik zentriert ist, also beispielsweise der Computer oder das Smartphone, sind für die Träger der Praktik nicht, bzw. nur mittelbar, als Artefakte in das soziale Geschehen einbringbar. Auch wenn neuere Medientheorien Medien mit Hilfe des Praktikenbegriffs $\mathrm{zu}$ fassen versuchen (Couldry 2004) und Mediatisierung als technisch co-motivierter Wandel von Kommunikationspraktiken mit dem Potenzial, die Sinnkonstruktion und Deutungsmuster in sozialen Gemeinschaften zu verändern, verstanden wird (Thomas/ Krotz 2008, S. 28f.), so scheint mir im vorliegenden Fall die Spezifik der untersuchten Praktik gerade in der Abwesenheit des Körpers zu liegen. Diese körperliche Abwesenheit ist für die untersuchten Praktiken insofern konstitutiv (bzw. 
begünstigt zumindest ihren Vollzug), als neben der Trauer über die Trennung auch die Scham der Verlassenen, mithin die Angst vor der Gefahr einer symbolischen Verletzung und Herabsetzung durch die Beurteilungsmacht der Anderen (Seidler 1995, S. 41) Emotionen sind, die traditionell durch Rückzug, Verhüllung und Vermeidung gekennzeichnet sind. Die Abwesenheit ist daher eine Ermöglichungsbedingung für die im Vollzug der Praktik stattfindende Entäußerung. Dies soll nicht heißen, dass andere körperlich-materielle Aspekte nicht auch vorhanden sind; sie sind im vorliegenden Fall aber aufgrund der mangelnden Möglichkeit, sie für die Interaktionspartner wahrnehmbar und so zum Gegenstand der Praktik zu machen, von geringerer Bedeutung.

Im Hinblick auf das Spannungsverhältnis von Routinisiertheit und Unberechenbarkeit liegt das Potenzial der datengeleiteten Analyse primär auf dem Gebiet der Identifizierung wiederkehrender Muster und Musterabfolgen, wie sie auch von Fiehler et al. (2014) als Bestandteile von Praktiken aufgefasst werden. Die Rekonstruktion der Vorgeformtheit macht freilich auch die Identifizierung von Abweichung möglich. Die datengeleitete Analyse sprachlicher Muster hat zudem das Potenzial, den Eigensinn der sprachlichen Oberfläche zuungunsten von Konzepten wie Souveränität und Zweckhaftigkeit stark zu machen. Sie ist damit an die Überlegungen zur impliziten Logik von Praktiken anschließbar. Der Blick auf den Forschungsgegenstand durch die Brille rekurrenter sprachlicher Muster, die sich aus induktiven Analysen ergeben, lässt die diskursive Prägung jeder Äußerung hervortreten sowie die mit den sprachlichen Routinen vermittelten Intentionen und Emotionen.

\section{Praktiken Modellieren: Trostdialoge im Trennungsforum}

\subsection{Korpus und Methode}

Wie bereits angedeutet, beschäftigt sich die folgende Analyse exemplarisch mit der Modellierung der Praktik des Trostdialogs in Online-Diskussionsforen. Das zugrunde liegende Korpus wurde aus Texten auf der Website www.trennungforum.de zusammengestellt. Dabei handelt es sich um ein Informationsportal rund um die Themen Trennung, Scheidung und Liebeskummer. Die Website umfasst auch ein Forum, in dem die Autorinnen und Autoren sich gegenseitig austauschen können. Das Korpus besteht aus den Diskussionssträngen des Unterforums „Trennung“, in dem Personen ihre fast immer frischen Trennungserlebnisse 
erzählen und andere Userinnen und User um Trost und Rat bitten und diese anderen Userinnen und User kommentieren dann auch. Typische Namen für einzelne Diskussionsstränge sind „Bin wirklich verzweifelt!!!“, „Ratlosigkeit.....“, „Ich weiß nicht weiter....“, „Die totale Einsamkeit“, „Die größte Verzweiflung im Leben“, „Ausgetauscht!“ oder „Ich bin neu hier und furchtbar traurig“. Das Korpus umfasst 1.597 Threads aus den Jahren 2007 bis 2010 mit insgesamt 4.210.813 laufenden Wortformen und wurde mit dem TreeTagger lemmatisiert (Schmid 1995). Die Wahl des Korpus ist aus modelltheoretischer Sicht (vgl. Abschnitt 3) die operationale Abgrenzung des Gegenstandsbereichs, d.h. die Nichtberücksichtigung irrelevanter Original-Objekte.

Eine informierte Entscheidung über die Art der Reduktion als der Operation des Weglassens von Objektdetails, die dem pragmatischen Zweck, den das Modell erfüllen soll, nicht dienlich sind, ist nur vor dem Hintergrund einer Funktionsbeschreibung für das Modell möglich. Das in diesem Beitrag verfolgte Ziel ist der Entwurf eines Modells für Trostdialoge, das es erlaubt, die Tätigkeiten, die von den Trägern der Praktik vollzogen werden, zu simulieren, um von dort Rückschlüsse darauf zu ziehen, auf welche Weise der Vollzug der Praktik die Konstruktion sozialen Sinns ermöglicht. Die Passgenauigkeit, aber auch die Schwächen des Simulationsmodells sollen zudem daraufhin befragt werden, inwiefern sie zur Theoriebildung in der Interaktionalen Linguistik bzw. der maschinellen Dialogmodellierung beitragen können. Da wie oben beschrieben die Mediatisierung im vorliegenden Fall die materiellen Aspekte der Praktik unsichtbar macht, sollen die Produktionsbedingungen und Kontexte der Tätigkeiten, soweit sie nicht technischer Natur sind, nicht im Modell berücksichtigt werden. Ebenfalls nicht berücksichtigt werden die zeitlichen Dimensionen der Online-Dialoge: Eröffnende Turns eines Diskussionsstrangs und die Antworten darauf sind durch einen Phasenverzug gekennzeichnet, der sich zwar quantifizieren ließe, jedoch in seiner spezifischen Ausprägung noch nicht theoretisiert, geschweige denn empirisch untersucht worden ist. Hingegen sollen die Dimensionen der Interaktivität, der Prozessualität, der Methodizität und der Pragmatizität mehr oder weniger in das Modell integriert werden.

Das Modell soll also die Wechselseitigkeit der Beiträge berücksichtigen (Interaktivität). Dies soll dadurch geschehen, dass die jeweiligen Turns analytisch als Einheiten behandelt werden sollen. Dabei sollen allerdings vereinfachend alle Antworten der tröstenden und ratgebenden Personen, die auf einen Turn des Initiators bzw. der Initiatorin des jeweiligen Stranges folgen, zu einem Turn zusammengefasst werden. Es kommt nämlich häufiger vor, dass auf ein Posting des Initiators zwei oder mehr Antworten erfolgen, ehe sich der Initiator wieder in die Diskussion einschaltet und auf die Trost-Turns meist gesamthaft Bezug nimmt. 
Eine exakte Modellierung der einzelnen Dialogschritte wäre überaus schwierig und müsste auf deutlich mehr Daten zurückgreifen. Daher wurde aus pragmatischen Gründen entschieden, die Tätigkeiten des Trost Suchenden und der Trost Spendenden als analytische Einheiten anzusehen. Das Modell soll zudem die Abfolge von Aktivitäten berücksichtigen (Prozessualität), indem es innerhalb der Turns Musterabfolgen identifiziert und auch die Distribution der Muster abhängig von der Abfolge der Turns abbildet. Methodizität und Pragmatizität sollen modelliert werden, indem die Analyse anhand sprachlicher Oberflächenphänomene erfolgt, deren Musterhaftigkeit geeignet ist, als Kontextualisierungshinweise für Sprachhandlungen und Modalitäten gelesen zu werden. Für die Messung dieser sprachlichen Muster wird auf eine Taxonomie von Sprachhandlungen zurückgegriffen, die für handlunsgtheoretisch fundierte Ansätze im Bereich Deutsch als Fremdsprache entwickelt wurde (Glaboniat et al. 2005). Diese Taxonomie, die in Abbildung 1 als hierarchischer Graph visualisiert ist, ordnet den einzelnen Sprechakttypen auch sprachliche Muster zu. Diese sprachlichen Muster wurden für die vorliegende Analyse in ein maschinell verarbeitbares Format gebracht und für Forendaten behutsam ergänzt. Die Sprachhandlung BEDAUERN etwa ist in der Taxonomie eine Unterkategorie von „Beurteilung von Zuständen, Ereignissen, Handlungen“, die wiederum der Oberkategorie „Bewertung, Kommentar“ zugeordnet ist. BEDAUERN werden sprachliche Muster wie „schade!“, „es ist schade, dass“, „es tut mir Leid“, „was für ein Pech“, „Ich bedaure“ oder „wenn ich gewusst hätte“ zugeordnet. Die Modellierung von Praktiken mittels Sprachhandlungstypen scheint auf den ersten Blick widersprüchlich, wurden doch Praktiken gerade als nicht dominante intentionale Tätigkeiten bestimmt. Dem kann jedoch mit Verweis auf die Interpretation der Austin'schen Sprechakttheorie durch Sybille Krämer und Marco Stahlhut (Krämer/Stahlhut 2001, S. $38 \mathrm{f}$.) begegnet werden, die gerade nicht das souveräne Subjekt als ,intentionalen Ursprung und Zentrum seines Handelns“ ansehen, sondern den Vollzug von Sprachakten als interpersonal (nicht notwendigerweise intersubjektiv!) koordinierte Tätigkeiten deuten. An die Stelle eines Handlungsmodells tritt also in ihrer Deutung ein am Ritual orientiertes Modell, bei dem durch den gemeinschaftlichen Vollzug von ,Handlungen‘ die im Ritual konventionalisierten Normen und Intentionen von den Interagierenden aufgenommen und übernommen werden. Diese Deutung wiederum passt zum oben zitierten Subjektverständnis der Praxistheorie, in dem pragmatische Zwecke den Praktiken inhärent sind und von den Akteuren als individuelle Intentionen umgedeutet werden (Reckwitz 2003, S. 293). 


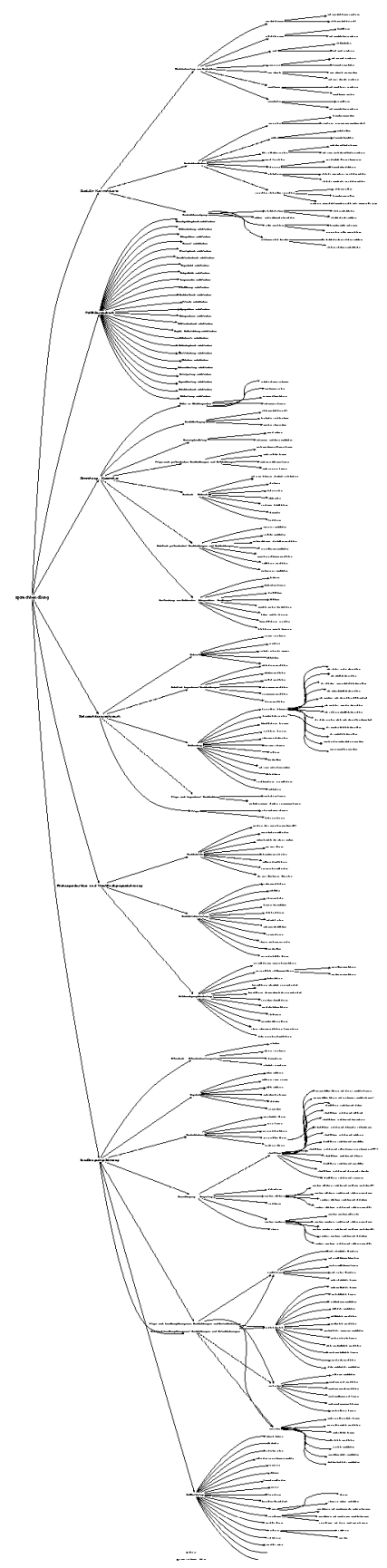

Abb. 1: Taxonomie der Sprechakte nach Glaboniat et al. (2005) 
Die Dekomposition als Operation der Zerlegung in einzelne Segmente im Prozess der Modellierung erfolgt mittels eines Mapping der Sprachhandlungstypen auf die jeweiligen Turns mit Hilfe der sprachlichen Muster aus Glaboniat et al. (2005). Im Folgenden findet sich ein Beispiel für einen mit Sprachhandlungstypen annotierten Turn:

\footnotetext{
$<$ speechact type $=$ "Anrede" pattern $=$ "Hallo" $>$ Hallo $</$ speechact $>\star \star \star !$ Auch $<$ speechact type $=$ "Mitgefühl ausdrücken" pattern="mir tut es .+? leid">mir tut es sehr leid</speechact $>$, wieviel und was Dir da passiert ist!!! <speechact type="Informationen erfragen" pattern= "Was .+? \?">Was mich persönlich sehr interessiert, ist, wie das aussah: Der Rosenkrieg in bezug auf Deine neue Liebe? $</$ speechact $><$ speechact type="Informationen erfragen" pattern="Was .+? \?">Was hat es denn so schwer gemacht?</speechact $>$ Und wie lässt sich das Verhalten Deines Mannes denn erklären, da ER doch damals wegen einer neuen Liebe gegangen war?! Ich habe einen sehr machthungrigen Noch-Mann, der aber mittlerweile etwas "zahmer" zu werden scheint, weil ich ihm die Stirn biete...! Also, <speechact type= "Dinge anbieten" pattern = "wenn Du magst" $>$ wenn Du magst $</$ speechact $>$, schreib Dir das alles von der Seele!
}

Die Sprachhandlungen ANREDE, MITGEFÜHL AUSDRÜCKEN, ERFRAGEN VON INFORMATIONEN und DINGE ANBIETEN wurden hier automatisiert erkannt und annotiert.

Die für den Modellierungsprozess zentrale Operation der Aggregation soll in mehreren Schritten erfolgen. Erstens soll anhand der Distribution der einzelnen Sprechakttypen ermittelt werden, welche Sprachhandlungen für die jeweiligen Turns typisch sind. Darüber hinaus soll die typische Abfolge von Sprechakten innerhalb eines Turns mittels der durchschnittlichen Position ihres Auftretens berechnet werden, um typische Adjazenzbeziehungen zwischen Mustern, die für die Praktik konstitutiv sind, zu ermitteln.

Um aus dem so konstruierten stochastischen Modell ein Simulationsmodell zu machen, mit dessen Hilfe die Praktik in unterschiedlichen Varianten generiert werden kann bzw. mit Hilfe dessen ein Vollzug der Praktik als Interaktion zwischen Computer und Mensch möglich ist, bedarf es zudem einer Extraktion von Satzmustern, mit deren Hilfe die jeweiligen Sprachhandlungstypen grammatikalisch korrekt und inhaltlich kohärent konstruiert werden können. Hierfür wurden auch semantische Kategorien aus GermaNet (Hamp/Feldweg 1997) und Dornseiff/Quasthoff/Wiegand (2004) verwendet.

\subsection{Analyse und Modellierung}

Die Analyse der Initialturns eines Diskussionsstrangs, also jener Turns, in denen die Trost und Rat Suchenden ihre Anfragen an die Online-Community stellen, ergab das 
in Abbildung 2 dargestellte Sequenzmuster für die vorkommenden Sprachhandlungstypen. Die Schriftgröße repräsentiert dabei die Frequenz der einzelnen Sprachhandlungstypen, die zwischen 1.527 (95,6\% aller Initialturns) im Fall der ANREDE und $168(10,5 \%)$ im Fall von KUMMER AUSDRÜCKEN variiert. Sprechakttypen, die in weniger als 10\% des entsprechenden Turns aller Threads vorkamen, wurden nicht berücksichtigt. Die Abfolge der Sprechakttypen ergibt sich aus dem Durchschnitt des relativen Vorkommens der Sprachhandlung in den Texten. Parallel angeführte Sprachhandlungstypen treten durchschnittlich an ähnlichen Positionen im Text auf.

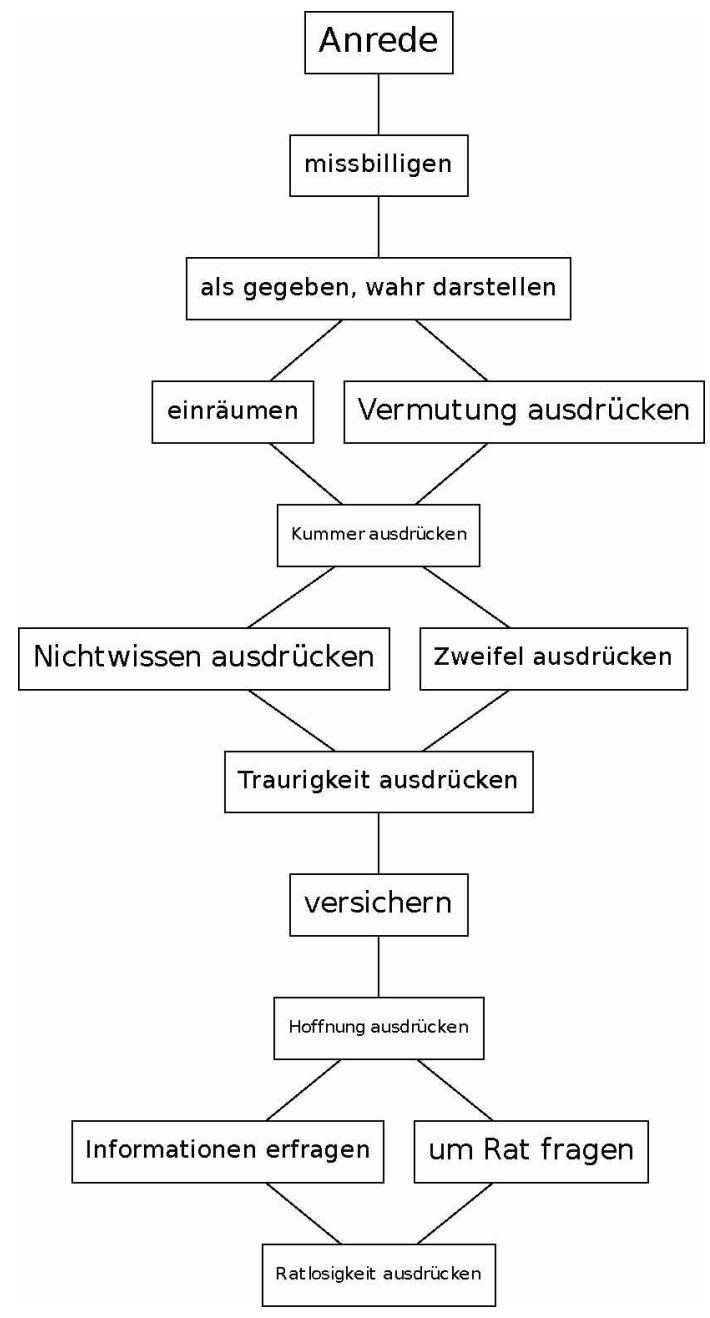

Abb. 2: Sequenzmuster der Sprachhandlungstypen in Inititalturns in trennung-forum.de 
Die Analyse zeigt, dass die Praktik des Trostdialogs durch einen Turn eröffnet wird, in dem nach der Anrede zunächst eine negative Einstellung zum Ausdruck gebracht wird (MISSBILLIGEN), ehe Faktisches berichtet (ALS GEGEBEN, WAHR DARSTELLEN) und - man darf mutmaßen - über die Ursachen spekuliert wird (VERMUTUNG AUSDRÜCKEN). Es folgt der Ausdruck von Ratlosigkeit (NICHTWISSEN AUSDRÜCKEN), die Referenz auf eine Emotion, die in einem Verlust ihre Ursache hat (TRAUER) und das VERSICHERN. Zuletzt (selten eingeleitet durch den Ausdruck von Hoffnung oder gefolgt vom Ausdruck der Ratlosigkeit) folgen die Sprachhandlungen UM RAT FRAGEN oder INFORMATIONEN ERFRAGEN.

Der Initialturn konstruiert die Autorin bzw. den Autor somit als orientierungslos (VERMUTUNG, NICHTWISSEN, ZWEIFEL, UM RAT FRAGEN) und motiviert daraus die Frage um Rat, mit der als übergaberelevante Stelle der Turn endet. Interessant ist, dass der Faktizitätsdarstellung zu Beginn des Turns noch eine negative Evaluation vorangestellt wird, obwohl die Bewertung eigentlich schon durch den Äußerungskontext und das beschriebene Faktum klar ist. Die Anrede in 95,6\% der Initialturns zeigt, dass eine Gemeinschaft adressiert und evoziert wird; die Tatsache, dass keine Verabschiedung erfolgt, kann so gedeutet werden, dass eine Antwort tatsächlich erwartet wird und die Intention besteht, auf diese selbst erneut zu erwidern. Es geht den Initiatorinnen und Initiatoren der Stränge also nicht um bloße Selbstentäußerung.

Tatsächlich bleibt auch kein Initialturn ohne Antwort. Eine Analyse der auf den Initialturn folgenden Antworten bis zum nächsten Turn des Initiators/der Initiatorin des jeweiligen Threads offenbart auch hier typische Adjazenzbeziehungen zwischen den einzelnen Sprachhandlungstypen. Abbildung 3 zeigt, dass auf die Anrede zunächst Emotionsdisplays folgen: der AUSDRUCK VON MITGEFÜHL sowie der AUSDRUCK VON FREUDE oder TRAUER. In den folgenden Abschnitten wird NICHTWISSEN, ZWEIFEL ausgedrückt, werden VERMUTUNGEN angestellt und schließlich INFORMATIONEN erfragt, ergänzt lediglich durch Ausdrücke des Lobs oder der Missbilligung. Die logische Modalität der Faktizität rückt also in den Vordergrund. Es folgen die Sprachhandlungstypen des RAT GEBEN, EMPFEHLEN oder ABRATEN und der Ausdruck von Eventualität (ALS MÖGLICH DARSTELLEN). Schließlich steht die Übertragung positiver Gefühle auf die Initiatoren bzw. den Initiator im Zentrum der Texte (HOFFNUNG AUSDRÜCKEN, ERMUNTERN, GUTE WÜNSCHE AUSSPRECHEN), ehe der Turn mit einer Verabschiedung beendet wird. 


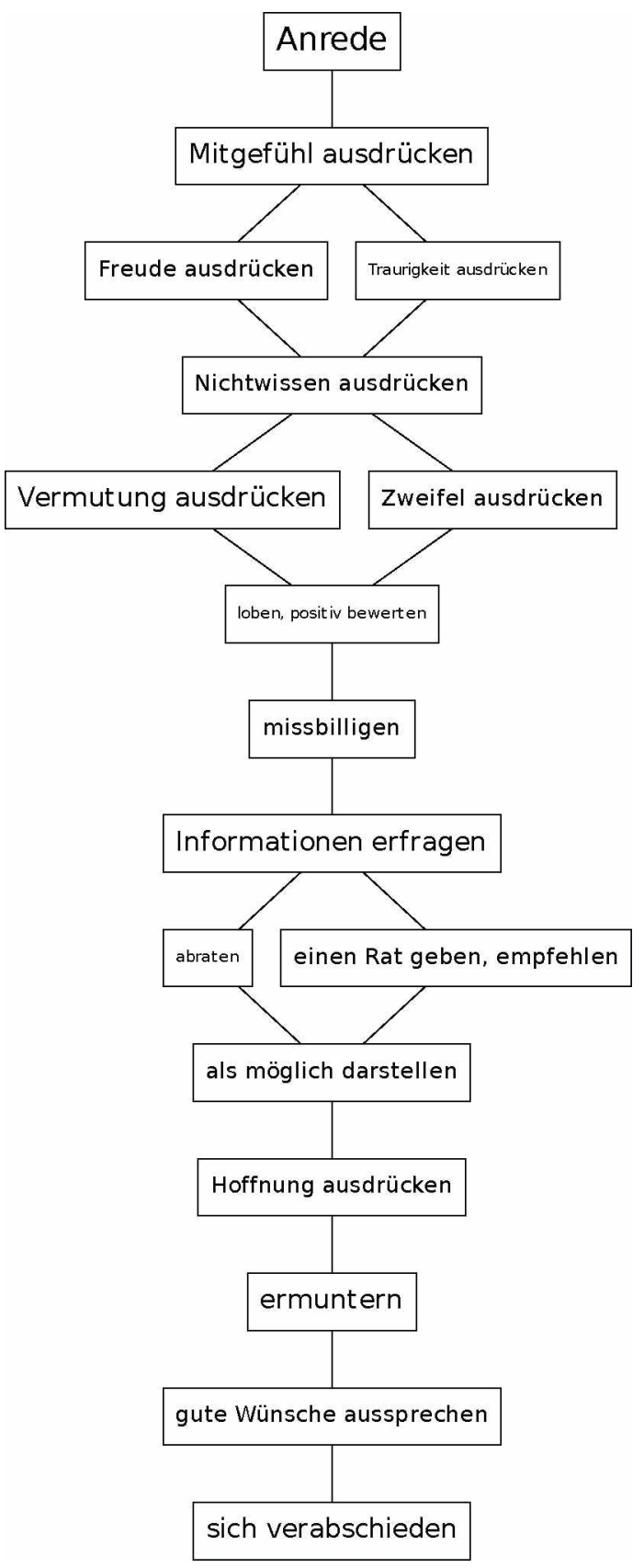

Abb. 3: Sequenzmuster der Sprachhandlungstypen in ersten Antwortturns in trennung-forum.de 
Interessant an diesem Befund ist, dass die Verhandlung von Wissen und damit die Herstellung von Faktizität ein zentraler Bestandteil der Antwort ist, die im Text vor dem Ratgeben stattfindet. Der Rat muss also epistemisch begründet oder zumindest durch Wissen angeleitet sein, damit die erwünschten positiven Effekte eintreten. Die zentrale Bedeutung der Herstellung von Faktizität zeigt sich auch, wenn man weitere Turns in die Analyse einbezieht (Abbildung 4).

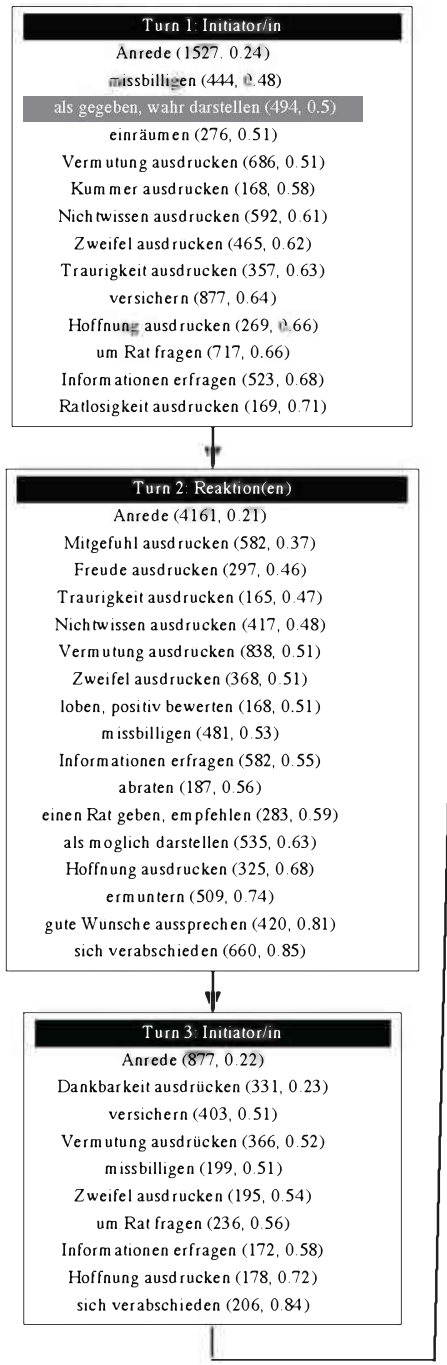

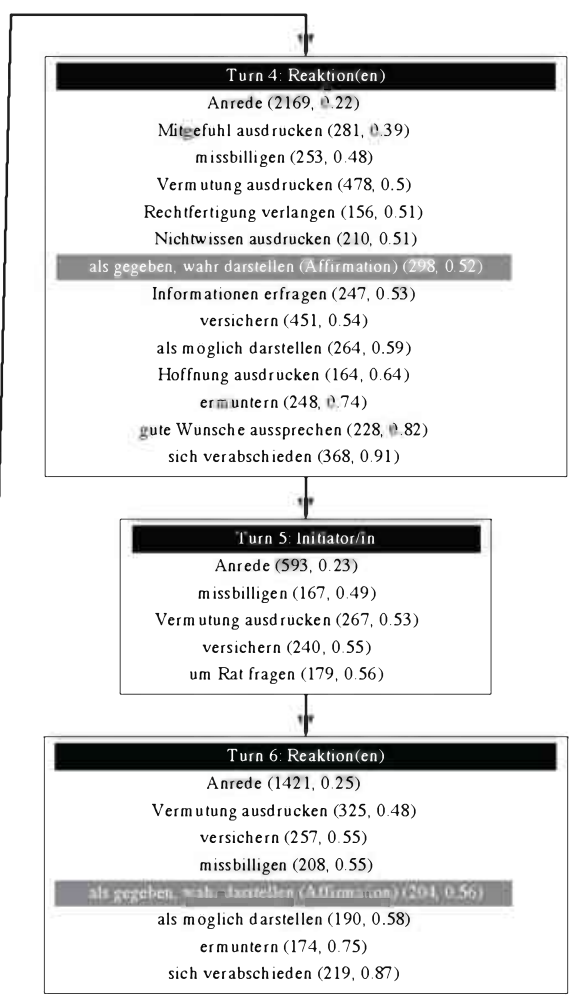

Abb. 4: Sequenzmuster der Sprachhandlungstypen in den ersten sechs Turns der Threads in trennung-forum.de 
Ab Turn 4 finden sich in den Reaktionsturns verhältnismäßig häufiger Sprechakte vom Typ ALS GEGEBEN, WAHR DARSTELLEN, während dieser Sprechakttyp bei den Turns der Initiatorin/des Initiators nach dem Initialturn nicht mehr in nennenswerter Frequenz auftritt. Für die Praktik des Trostgesprächs ist es also konstitutiv, dass die Herstellung von Faktizität vom Initiator auf den Ratenden und Tröstenden übertragen wird.

Auf der Basis dieses statischen Modells der Praktik ist es möglich, ein Simulationsmodell zu entwickeln. Hierfür müssen die Sprachhandlungstypen in satzwertige Sprachhandlungen übertragen werden. Das oben vorgeschlagene Modell sieht vor, Sätze wie Frames mit Slots aufzufassen, die, restringiert durch statistisch ermittelte morphosyntaktische und semantische Regeln, gefüllt werden. Ein stark vereinfachtes Modell eines Satzes, der den Sprachhandlungstyp TRAUER AUSDRÜCKEN repräsentiert, sieht dann etwa so aus:

\begin{tabular}{|c|c|c|c|c|c|}
\hline & Intensivierer & $\begin{array}{l}\text { Emotionswort } \\
\text { Trauer }\end{array}$ & $\begin{array}{l}\text { Possesiv- } \\
\text { pronomen }\end{array}$ & $\begin{array}{l}\text { Bezeichnung } \\
\text { für Partner }\end{array}$ & \\
\hline \multirow[t]{5}{*}{ Ich bin } & ADJD & ADJD & , PPOSAT & NN & hat mich verlassen \\
\hline & sehr & traurig & mein & Freund & \\
\hline & total & fertig & meine & Mann & \\
\hline & unheimlich & down & & Macker & \\
\hline & furchtbar & deprimiert & & Liebe & \\
\hline
\end{tabular}

Satzmuster und Filler sind datengeleitet ermittelt (Filler hier freilich nur im Ausschnitt wiedergegeben); die Filler kookkurrieren in spezifischer Weise. Mit Hilfe dieser Satzmodelle ist es möglich, für die meisten der Sprachhandlungstypen wohlgeformte Sätze maschinell zu generieren. Ein maschinell generierter Initialturn sieht dann beispielsweise so aus:

\footnotetext{
Hallo!

Es ist so: vor 2 Tagen hat mein Freund die Beziehung beendet.

Ich vermute, er hat eine Neue, denn er war vorher schon komisch.

Ich weiß nicht, was ich machen soll.

Ich könnte den ganzen Tag nur heulen.

Es ist wirklich so schwer, mit der Trennung zu leben.

Was soll ich tun?
}

Mangelnde Detaillierung und geringe Kohärenz lassen zwar den Text etwas merkwürdig erscheinen. Ob und zu welchem Grad derartig hergestellte Texte sich als 
Initialturns eignen, kann jedoch erst anhand der Reaktionen entschieden werden. Eine Simulierung der Reaktionen ist freilich ungleich schwerer, weil die zentrale Handlung des Ratgebens aus Akten der Faktizitätsherstellung motiviert werden muss. Dies ist jedoch ohne Rückgriff auf Wissensressourcen nicht zu leisten. In jedem Fall aber hätte das Simulationsmodell das Potenzial, durch gezieltes Hinzufügen oder Weglassen einzelner Sprachhandlungstypen deren konstitutive Funktion in einem Feldexperiment zu testen. Auf diese Weise kann das Modell der Praktik des Trostdialogs stetig verbessert werden. ${ }^{4}$

\section{Fazit: Mensch und Maschine im Austausch}

Die Dialogmodellierung hat in den letzten Jahren so erhebliche Fortschritte gemacht, dass sie auch von der Interaktionalen Linguistik als Methode genutzt werden könnte. Hierfür wäre eine Öffnung der Interaktionalen Linguistik hin zu modellorientierter Forschungslogik und datengeleiteten Methoden hilfreich. Der vorliegende Beitrag hat lediglich einen möglichen Zugang zur Analyse von Praktiken skizziert. Agentenbasierte Systeme, die nicht notwendigerweise natürlichsprachlich kommunizieren, könnten ebenfalls zur Simulation bestimmter Eigenschaften sprachlicher Interaktionen benutzt werden.

Die Verbreitung von Dialogsystemen wird freilich auch nicht ohne Folgen für die Gestaltung von verbaler Interaktion insgesamt bleiben; denn Dialogsysteme zwängen Menschen in das Prokrustesbett ihrer Kategorien, wenn diese erfolgreich mit Maschinen kommunizieren wollen. Die langfristige Konsequenz könnte sein, dass Praktiken zum einen stärker normiert werden und dass zum anderen die an der Interaktion Beteiligten mehr als bisher ihre im Medium der Sprache vollzogenen Handlungen und ihre Interpretation der Situation explizit zum Ausdruck bringen, um Missverständnisse zu vermeiden. Und obwohl die Dialogmodellierung anstrebt, Maschinen wie Menschen kommunizieren zu lassen, werden Menschen bestrebt sein, eine ,anthropologische Differenz' durch Praktiken der verbalen Interaktion herzustellen.

Je mehr Dialogsysteme in unserem Alltag zur Anwendung kommen, desto wichtiger wird also die Beschäftigung mit ihnen auch aus kulturanalytischer Sicht. Clifford Geertz bestimmte die Aufgabe der Kulturanthroplogie wie folgt:

4 Freilich müssen hier auch persönlichkeits- und datenschutzrechtliche sowie forschungsethische Grenzen berücksichtigt werden. Anonymisierung und informierte Zustimmung als Grundprinzipien müssen als Mindeststandards bei Experimenten, die mit Simulationen arbeiten, ihre Geltung behalten. 
We are not, or at least I am not, seeking either to become natives [...] or to mimic them. Only romantics or spies would seem to find point in that. We are seeking, in the widened sense of the term in which it encompasses very much more than talk, to converse with them, a matter a great deal more difficult, and not only with strangers, than is commonly recognized. [...] Looked at in this way, the aim of anthropology is the enlargement of the universe of human discourse. (Geertz 1973, S. 13)

Und so wird uns ELIZA immer fragen und auffordern: „How do you do. Please tell me your problem.“

\section{Literatur}

Anderson, Chris (2008): The end of theory: The data deluge makes the scientific method obsolete. In: Wired Magazine 16.07 (23.6.2008). Internet: http://archive.wired.com/ science/discoveries/magazine/16-07/pb_theory/ (Stand: 15.6.2015).

Belica, Cyril (1996): Analysis of temporal changes in corpora. In: International Journal of Corpus Linguistics 1, S. 61-73.

Belica, Cyril (2001): Kookkurrenzdatenbank CCDB. Eine korpuslinguistische Denk- und Experimentierplattform für die Erforschung und theoretische Begründung von systemischstrukturellen Eigenschaften von Kohäsionsrelationen zwischen den Konstituenten des Sprachgebrauchs. Mannheim.

Bubenhofer, Noah (2009): Sprachgebrauchsmuster. Korpuslinguistik als Methode der Diskursund Kulturanalyse. (= Sprache und Wissen 4). Berlin/New York.

Colby, Kenneth Mark/Watt, James B./Gilbert, John P. (1966): A computer method for psychotherapy: Preliminary communication. In: Journal of Nervous and Mental Disease 142, S. $148-152$.

Couldry, Nick (2004): Theorising media as practice. In: Social Semiotics 14, S. 115-132.

Deppermann, Arnulf (2008): Gespräche analysieren: Eine Einführung. 4. Aufl. (= Qualitative Sozialforschung 3). Wiesbaden.

Dornseiff, Franz/Quasthoff, Uwe/Wiegand, Herbert Ernst (2004): Der deutsche Wortschatz nach Sachgruppen. 8., völlig neu bearb. und mit einem vollst. alphabet. Zugriffsregister versehene Aufl. Berlin.

Fiehler, Reinhard et al. (2004): Eigenschaften gesprochener Sprache. (= Studien zur Deutschen Sprache 30). Tübingen.

Geertz, Clifford (1973): The interpretation of cultures: Selected essays. (= Harper Torchbooks 5043). New York.

Glaboniat, Manuela et al. (2005): Profile deutsch. Berlin.

Hamp, Birgit/Feldweg, Helmut (1997): GermaNet - a Lexical-Semantic Net for German. In: Proceedings of ACL Workshop Automatic Information Extraction and Building of Lexical Semantic Resources for NLP Applications, 12. Juli 1997, Madrid. Somerset, NJ, S. 9-15. Hausendorf, Heiko (1997): Konstruktivistische Rekonstruktion. Theoretische und empirische Implikationen aus konversationsanalytischer Sicht. In: Sutter, Tilmann (Hg.): Beobachtung verstehen, Verstehen beobachten. Perspektiven einer konstruktivistischen Hermeneutik. Opladen, S. 254-272. 
Jokinen, Kristiina (2009): Constructive dialogue modelling: Speech interaction and rational agents. Chichester.

Krämer, Sybille/Stahlhut, Marco (2001): Das „Performative“ als Thema der Sprach- und Kulturphilosophie. In: Fischer-Lichte, Erika/Wulf, Christoph (Hg.): Theorien des Performativen. (= Paragrana 1). Berlin, S. 35-64.

Kühne, Thomas (2005): What is a Model? In: Bézivin, Jean/Heckel, Reiko (Hg.): Language engineering for model-driven software development. Dagstuhl Seminar Proceedings 04101, February 29th to April 5th 2004.

Perkuhn, Rainer/Belica, Cyril (2006): Korpuslinguistik - das unbekannte Wesen oder Mythen über Korpora und Korpuslinguistik. In: Sprachreport 22/1, S. 2-8.

Popper, Karl Raimund (1966): Logik der Forschung. 2., erw. Aufl. (= Die Einheit der Gesellschaftswissenschaften 4). Tübingen.

Reckwitz, Andreas (2003): Grundelemente einer Theorie sozialer Praktiken. Eine sozialtheoretische Perspektive. In: Zeitschrift für Soziologie 32, S. 282-301.

Reckwitz, Andreas (2008): Unscharfe Grenzen. Perspektiven der Kultursoziologie. Bielefeld.

Schatzki, Theodor R./Knorr Cetina, Karin/von Savigny, Eike (Hg.) (2001): The practice turn in contemporary theory. London.

Scharloth, Joachim/Eugster, David/Bubenhofer, Noah (2013): Das Wuchern der Rhizome. Linguistische Diskursanalyse und Data-driven Turn. In: Busse, Dietrich/Teubert, Wolfgang $(\mathrm{Hg}$.): Linguistische Diskursanalyse. Neue Perspektiven. (= Interdisziplinäre Diskursforschung). Wiesbaden, S. 345-380.

Schmid, Helmut (1995): Improvements in part-of-speech tagging with an application to German. In: Proceedings of the EACL'95 SIGDAT Workshop. Dublin, S. 47-50.

Schütte, Reinhard (1998): Grundsätze ordnungsmäßiger Referenzmodellierung: Konstruktion konfigurations- und anpassungsorientierter Modelle. (= Neue Betriebswirtschaftliche Forschung 233). Wiesbaden.

Seidler, Günter (1995): Der Blick des Anderen - eine Analyse der Scham. Stuttgart.

Sinclair, John (1991): Corpus, concordance, collocation. (= Describing English Language). Oxford. Stachowiak, Herbert (1973): Allgemeine Modelltheorie. Wien/New York, S. 131-134.

Stachowiak, Herbert (1983): Erkenntnisstufen zum Systematischen Neopragmatismus und zur Allgemeinen Modelltheorie. In: Stachowiak, Herbert (Hg.): Modelle - Konstruktion der Wirklichkeit. (= Kritische Information 101). München, S. 87-146.

Steyer, Kathrin (2004): Kookkurrenz. Korpusmethodik, linguistisches Modell, lexikografische Perspektiven. In: Steyer, Kathrin (Hg.): Wortverbindungen - mehr oder weniger fest. (= Jahrbuch des Instituts für Deutsche Sprache 2003). Berlin/New York, S. 87-116.

Steyer, Kathrin/Brunner, Annelen (2009): Das UWV-Analysemodell. Eine korpusgesteuerte Methode zur linguistischen Systematisierung von Wortverbindungen. (= OPAL 1/2009). Mannheim. Internet: http://pub.ids-mannheim.de/laufend/opal/opal09-1.html (Stand: 4.8.2015).

Steyer, Kathrin/Lauer, Meike (2007): Corpus-Driven. Linguistische Interpretation von Kookkurrenzbeziehungen. In: Eichinger, Ludwig M./Kämper, Heidrun (Hg.): Sprach-Perspektiven. Germanistische Linguistik und das Institut für Deutsche Sprache. (= Studien zur Deutschen Sprache 40). Tübingen, S. 493-509.

Thomas, Tanja/Krotz, Friedrich (2008): Medienkultur und Soziales Handeln: Begriffsarbeiten zur Theorieentwicklung. In: Thomas, Tanja (Hg.): Medienkultur und soziales Handeln. (=Medien - Kultur - Kommunikation). Wiesbaden, S. 17-42. 
Teubert, Wolfgang (2005): My version of corpus linguistics. In: International Journal of Corpus Linguistics 10, S. 1-13.

Tognini-Bonelli, Elena (2001): Corpus linguistics at work, studies in corpus linguistics. (= Studies in Corpus Linguistics 6). Amsterdam.

Weizenbaum, Joseph (1966): ELIZA - A computer program for the study of natural language communication between man and machine. In: Communications of the ACM 9, S. 36-45.

Weizenbaum, Joseph (1976): Computer power and human reason: From judgment to calculation. New York. 